\title{
Evaluation of the Forward-Backward Sweep Load Flow Method using the Contraction Mapping Principle
}

\author{
Diego Issicaba ${ }^{1}$ and Jorge Coelho ${ }^{2}$ \\ ${ }^{1}$ Department of Electrical Engineering, Federal University of Technology - Parana (UTFPR), Curitiba-PR, Brazil \\ ${ }^{2}$ Department of Electrical Engineering, Federal University of Santa Catarina (UFSC), Florianopolis-SC, Brazil
}

\begin{abstract}
Article Info
Article history:

Received May 19, 2016

Revised Jul 12, 2016

Accepted Aug 1, 2016

Keyword:

Power engineering

Power distribution systems

Load flow analysis
\end{abstract}

Convergence

\section{Corresponding Author:}

Diego Issicaba

Department of Electrical Engineering, Federal University of Technology - Parana (UTFPR)

Av. Sete de Setembro, 3165, Sector D, Rebouças, 80230-910 Curitiba-PR, Brazil

+55 $413310-4626$

issicaba@utfpr.edu.br

\section{INTRODUCTION}

Load flow methods are widely utilized in power system analysis and applications $[1,2,3]$. One reasonable way to understand and compare load flow approaches is through their mathematical convergence analysis. The challenges behind analyzing the convergence of iterative methods begin with the characterization of the problem solution itself. Some researches have studied the nature and multiplicity of stable load flow solutions for transmission systems $[4,5,6,7]$, and a few researches have studied the load flow solution for power distribution systems. H. D. Chiang and M. E. Baran [8] showed the existence and uniqueness of the feasible load flow solution for balanced distribution systems, while J. F. Chen and W. M. Wang [9] verified the existence and feasibility of the load flow solution by using a formulation based on the DistFlow equations $[10,11]$. In [12], K. N. Miu and H. D. Chiang provided a useful contribution by extending the work in [9] to the three-phase case with detailed network modeling.

Since an analytical solution does not exist for the load flow problem, even given some knowledge about the solution, it is necessary to formulate iterative procedures for the load flow calculation. The aforementioned procedures might guarantee a fast convergence towards a solution, given an initial estimate and a fixed tolerance. Hence, aiming at analyzing the convergence of load flow algorithms for transmission systems, J. Meisel and R. D. Barnard [13] presented a view of the Gauss-Seidel method and the Newton-Raphson method in terms of a fixed-point formulation. K. Ganesan et al. [14] studied the convergence of the Newton-Raphson method by using the Kantorovich theorem, and $\mathrm{F}$. Wu [15] proved the convergence and demonstrated the strong convergence dependence of network $r / x$ ratios for the Fast Decoupled method.

Regarding distribution system analysis, there is a lack of convergence formalization for load flow methods. In fact, these methods are not usually based on Jacobian evaluations and generally employ sweep procedures in which currents [16] (or powers [17, 18]) are accumulated from end nodes towards the substation bus. In this area, E. Bompard et al. [19] contributed significantly by studying the convergence of a sweep

Journal Homepage: http://iaesjournal.com/online/index.php/IJECE 
method and verifying that its convergence properties can be depreciated for high loadings. This observation was first brought out by R. P. Broadwater in his discussions of [16].

In this context, this paper presents the assessment the forward-backward sweep load flow method proposed by D. Shirmohammadi et al. [16] using fixed-point concepts and the contraction mapping theorem. Also, an alternative argument showing the existence and uniqueness, under certain conditions, of the load flow solution is presented. At last, a set of error boundaries per iteration limiting the vectorial distance between a voltage iterate values and the solution is deduced.

The paper is organized as follows. Section 2 presents a brief mathematical background of the contraction mapping theorem and the load flow method under assessment. In Section 3, the aforementioned mathematical contributions are demonstrated for a general distribution system. Numerical simulations are shown in Section 4 aiming at validating the theoretical results. Finally, Section 5 outlines conclusions and future works.

\section{MATHEMATICAL BACKGROUND}

\subsection{The Contraction Mapping Theorem}

The fixed-point theorems compose a set of theorems applied in several areas such as physics, mathematics, economics and engineering. We can define a fixed-point $\mathbf{x}^{\propto}$ of a function $\mathbf{\Phi}: \mathbf{X} \rightarrow C^{n}\left(\mathbf{X} \subset C^{n}\right)$ as any point $\mathbf{x}^{\propto} \in \mathbf{X}$ such that $\mathbf{\Phi}\left(\mathbf{x}^{\propto}\right)=\mathbf{x}^{\propto}$. Some of the most important fixed-point theorems are the Brouwer theorem, the Knaster-Tarski theorem, the Lefschetz theorem and the contraction mapping theorem utilized in this work. Fixed-point theorems have an important role in the analysis of nonlinear problems, as well as the analysis of algorithms associated to the solution of these problems.

With the aim of introducing the contraction mapping theorem, let us define a general nonlinear problem composed by $n$ nonlinear equations $f_{i}\left(\mathbf{x}_{1}, \ldots, \mathbf{x}_{n}\right)=y_{i}$, for all $i=1, \ldots, n$. For simplicity, note that this problem can also be represented in the vector form $\mathbf{F}(\mathbf{x})=\mathbf{y}$, where $\mathbf{x}=\left[x_{i}\right]_{n}$ and $\mathbf{y}=\left[y_{i}\right]_{n}$. By formulating iterative methods to solve this system of equations, we search for an iteration scheme $\mathbf{x}^{(k+1)}=\boldsymbol{\Phi}\left(\mathbf{x}^{(k)}\right)$ aiming at generating a sequence of iterates $\left\{\mathbf{x}^{(0)}, \mathbf{x}^{(1)}, \ldots\right\}$ that converges if and only if $\mathbf{F}(\mathbf{x})=\mathbf{y}$. One of the reasonable choices for this iteration scheme is

$$
\boldsymbol{\Phi}\left(\mathbf{x}^{(k)}\right)=\mathbf{x}^{(k)}-\mathbf{W}\left(\mathbf{x}^{(k)}\right)\left[\mathbf{F}\left(\mathbf{x}^{(k)}\right)-\mathbf{y}\right]
$$

where $\mathbf{W}(\mathbf{x})$ is a $n \times n$ nonsingular matrix function of $\mathbf{x}$. In the simple iteration scheme above, the convergence is obtained at a fixed-point and $\mathbf{x}^{\propto}$ is a fixed-point of $\mathbf{\Phi}$ if and only if $\mathbf{F}(\mathbf{x})=\mathbf{y}$.

We emphasize that once an iteration scheme is chosen, by starting with a point $\mathbf{x}^{(0)}$ the sequence of iterates must converge to a fixed-point $\mathbf{x}^{\propto}$, i.e. $\lim _{k \rightarrow \infty}|| \mathbf{x}^{(k)}-\mathbf{x}^{\propto} \|=0$, in a reasonable amount of time. There also exists an interesting property in which the mismatches between two subsequent iterates $\left\|\mathbf{x}^{(k+1)}-\mathbf{x}^{(k)}\right\|$ geometrically decrease throughout the iterative process. In mathematical terms this property can be written as

$$
\left\|\boldsymbol{\Phi}\left(\mathbf{x}^{(k+1)}\right)-\boldsymbol{\Phi}\left(\mathbf{x}^{(k)}\right)\right\| \leq c\left\|\mathbf{x}^{(k+1)}-\mathbf{x}^{(k)}\right\|
$$

for some $c \in R$ such that $0 \leq c<1$.

In a general sense, if a function $\boldsymbol{\Phi}$ satisfies the inequality $\left\|\Phi(\mathbf{x})-\mathbf{\Phi}\left(\mathbf{x}^{\prime}\right)\right\| \leq c\left\|\mathbf{x}-\mathbf{x}^{\prime}\right\|, \forall \mathbf{x}, \mathbf{x}^{\prime} \in \mathbf{X}$, for some $c \in R$ such that $0 \leq c<1$, then $\boldsymbol{\Phi}$ is a contraction mapping with contraction constant $c$. The contraction mappings have two interesting properties, both presented in the following theorem.

Theorem 1 (The Contraction Mapping Theorem) [20]: Let $\mathbf{X} \subset C^{n}$ be a closed subset and $\mathbf{\Phi}: \mathbf{X} \rightarrow \mathbf{X}$ be a map such that

$$
\left\|\boldsymbol{\Phi}(\mathbf{x})-\mathbf{\Phi}\left(\mathbf{x}^{\prime}\right)\right\| \leq c\left\|\mathbf{x}-\mathbf{x}^{\prime}\right\|
$$

for some contraction constant $0 \leq c<1, c \in R, \forall \mathbf{x}, \mathbf{x}^{\prime} \in \mathbf{X}$. Then $\boldsymbol{\Phi}$ has an unique fixed-point in $\mathbf{X}$. Additionally, the sequence of iterates $\left\{\mathbf{x}^{(0)}, \boldsymbol{\Phi}\left(\mathbf{x}^{(0)}\right), \boldsymbol{\Phi}\left(\mathbf{\Phi}\left(\mathbf{x}^{(0)}\right)\right), \ldots\right\}$ converges to the fixed-point for any $\mathbf{X}^{(0)} \in \mathbf{X}$.

This theorem will be used to analyze some important properties of the forward-backward sweep load flow method. It is important to emphasize that the nonlinear iteration scheme in (1) is the basis in which classical methods solve load flow equations for high voltage transmission systems. For instance, if we take $\mathbf{W}\left(\mathbf{x}^{(k)}\right)$ to be the inverse Jacobian matrix of the function $\mathbf{F}$ at $\mathbf{x}^{(k)}$, then (1) becomes the update rule of the Newton-Raphson method. On the other hand, in case of distribution system analysis, the update rules of the methods change considerably depending upon the chosen approach, although it is possible to establish some similarities to the nonlinear iteration scheme presented in (1).

IJECE Vol. 6, No. 6, December 2016: 3229 - 3237 


\subsection{The Forward-Backward Sweep Method}

With the aim of mathematically describing D. Shirmohammadi et al.'s load flow method [16], let us consider the radial schematic depicted in Fig. 1, where lines are modeled as series impedances $z_{i}$ while complex bus voltages and complex load demands are modeled, respectively, by $E_{i}=e_{i}+j f_{i}$ and $S_{i}, \forall i=1, \ldots, n$. The substation bus is named the 0 (zero) bus with complex voltage denoted by $E_{0}$. Observe that the index $i$ is used to identify the bus and the line upstream this bus, depending on the variables involved.

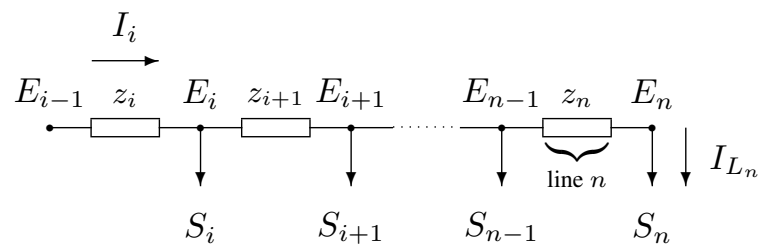

Figure 1. Radial distribution network schematic.

In distribution systems load flow analysis, the substation bus is usually assumed to be the slack bus, with a constant real voltage $E_{0}$. Under this assumption, the algorithm begins with an initial solution for all buses and performs three basic steps until a convergence criterion is satisfied.

1. The current injection $I_{L_{i}}^{(k+1)}$ at bus $i$ and iteration $k+1$ is calculated as $I_{L_{i}}^{(k+1)}=S_{i}^{*} / E_{i}^{*(k)}$, where $E_{i}^{(k)}$ is the complex voltage at bus $i$ calculated during the $k^{\text {th }}$ iteration;

2. Starting from the end branches and moving towards the branch connected to the substation bus, the current at branch $i$ can be calculated by $I_{i}^{(k+1)}=I_{L_{i}}^{(k+1)}+\sum_{r \in \Delta_{i}} I_{L_{r}}^{(k+1)}=\sum_{r \in \Lambda_{i}} I_{L_{r}}^{(k+1)}$, where $\Delta_{i}$ denotes the set of downstream buses of bus $i$, and $\Lambda_{i}$ denotes the set of elements of $\Delta_{i}$ including the $i^{\text {th }}$ bus, i.e. $\Lambda_{i} \triangleq \Delta_{i} \bigcup\{i\}$;

3. The bus voltages are updated in a forward sweep starting from the first branch and moving towards end branches by $E_{i}^{(k+1)}=E_{u_{i}}^{(k+1)}-z_{i} I_{i}^{(k+1)}$, where $u_{i}$ represents the upstream bus of the $i^{t h}$ bus.

One basic convergence criterion is the maximum absolute power mismatch for all buses. Alternatively, the convergence can be tested by other criteria like the maximum absolute difference between subsequent voltage iterates.

\section{CONTRACTION MAPPING ASSESSMENT}

\subsection{Convergence Rate}

Let us examine the convergence of complex voltages using the iterative scheme of the algorithm. Observe that the general update rule can be written recursively for iteration $k$ as

$$
\phi_{i}\left(E_{i}^{(k)}\right)=\phi_{u_{i}}\left(E_{u_{i}}^{(k)}\right)-z_{i} \sum_{r \in \Lambda_{i}}\left(\frac{S_{r}^{*}}{E_{r}^{*(k)}}\right), \quad \phi_{i}\left(E_{i}^{(k)}\right)=E_{i}^{(k+1)}
$$

Now let $p_{i}$ be the set of lines between the substation bus and bus $i$. Also, let $o_{i r}$ be the intersection of sets $p_{i}$ and $p_{r}$. Then, expression (4) can be rewritten as

$$
\phi_{i}\left(E_{i}^{(k)}\right)=E_{0}-\sum_{r=1}^{n} z_{i r}\left(\frac{S_{r}^{*}}{E_{r}^{*(k)}}\right), \quad z_{i r}=\sum_{t \in o_{i r}} z_{t}
$$

As a matter of consequence, the same rule can now be written for iteration $k+1$, such that the difference between subsequent voltage iterates can be recursively calculated as follows

$$
\Delta \phi_{i}^{(k+1)}=\sum_{r=1}^{n} z_{i r} S_{r}^{*}\left(\frac{\Delta E_{r}^{*(k+1)}}{E_{r}^{*(k+1)} E_{r}^{*(k)}}\right)
$$


By rearranging the terms of the expression above, we can rewrite (6) in the convenient form

$$
\Delta \phi_{i}^{(k+1)}=\sum_{r=1}^{n} d_{i r}^{(k+1)} \Delta E_{r}^{*(k+1)}, \quad d_{i r}^{(k+1)}=\frac{z_{i r} S_{r}^{*}}{E_{r}^{*(k+1)} E_{r}^{*(k)}}
$$

As consequence, (7) can be expressed in their matrix forms

$$
\boldsymbol{\Delta} \boldsymbol{\Phi}^{(k+1)}=\mathbf{D}^{(k+1)} \boldsymbol{\Delta} \mathbf{E}^{*(k+1)}, \quad \mathbf{D}^{(k+1)}=\mathbf{Z S}^{*} \mathbf{F}^{(k+1)}
$$

where $\boldsymbol{\Phi}\left(\mathbf{E}^{(k)}\right)$ is a $n \times 1$ vector whose elements are the complex voltages obtained by the update rule at the $k^{t h}$ iteration, $\boldsymbol{\Delta} \boldsymbol{\Phi}^{(k+1)} \triangleq \boldsymbol{\Phi}\left(\mathbf{E}^{(k+1)}\right)-\boldsymbol{\Phi}\left(\mathbf{E}^{(k)}\right), \mathbf{E}^{(k)}$ is a $n \times 1$ vector whose elements are the complex voltages at the $k^{t h}$ iteration, $\Delta \mathbf{E}^{(k+1)} \triangleq \mathbf{E}^{(k+1)}-\mathbf{E}^{(k)}, \mathbf{Z}$ is the impedance matrix of the distribution network with size $n \times n, \mathbf{S}^{*}$ is a diagonal matrix with size $n \times n$ whose elements are the conjugated complex loads, and $\mathbf{F}^{(k+1)}$ is a diagonal matrix with size $n \times n$ whose elements are reciprocals of the product of subsequent conjugated complex voltage iterates. By applying the norm operator (in $C^{n}$ ) and their properties in (8) we have

$$
\left\|\boldsymbol{\Delta} \boldsymbol{\Phi}^{(k+1)}\right\| \leq\left\|\mathbf{D}^{(k+1)}\right\|\left\|\boldsymbol{\Delta} \mathbf{E}^{*(k+1)}\right\|=\left\|\mathbf{D}^{(k+1)}\right\|\left\|\boldsymbol{\Delta} \mathbf{E}^{(k+1)}\right\|
$$

At this point, different norms can be used to evaluate the convergence of the algorithm. In particular, we can evaluate $\left\|\mathbf{D}^{(k+1)}\right\|$ using the infinity norm (or Chebyshev norm) as follows.

$$
\left\|\mathbf{D}^{(k+1)}\right\|=\max _{1 \leq i \leq n} \sum_{r=1}^{n}\left\|d_{i r}^{(k+1)}\right\|=\sum_{r=1}^{n}\left\|\frac{z_{\hbar r} S_{r}^{*}}{E_{r}^{*(k+1)} E_{r}^{*(k)}}\right\|, \hbar=\arg \max _{1 \leq i \leq n}\left\{\sum_{r=1}^{n}\left\|\frac{z_{i r} S_{r}^{*}}{E_{r}^{*(k+1)} E_{r}^{*(k)}}\right\|\right\}
$$

Hence, inequality (9) can be conveniently written as

$$
\left\|\boldsymbol{\Delta} \boldsymbol{\Phi}^{(k+1)}\right\| \leq c^{(k+1)}\left\|\boldsymbol{\Delta} \mathbf{E}^{(k+1)}\right\|, \quad c^{(k+1)}=\sum_{r=1}^{n}\left\|\frac{z_{\hbar r} S_{r}^{*}}{E_{r}^{*(k+1)} E_{r}^{*(k)}}\right\|
$$

Therefore, by using the inequalities above, the existence as well as the uniqueness of the feasible solution can be analyzed. Also, convergence properties of the load flow algorithm can be assessed and error boundaries per iteration specified.

\subsection{Existence and Uniqueness of the Feasible Solution}

The existence and uniqueness of the load flow feasible solution in closed subset is an important result for system analysis. Once this result is verified, it is guaranteed that studies such as reactive power compensation, distribution automation, and network reconfiguration, all usually endorsed by load flow calculations, will lead to the steady state obtained in practice, given the uncertainties of loading and network data.

Aiming at verifying the existence and uniqueness of the feasible load flow solution, let $\mathcal{R}$ be a closed subset of $C^{n}$ given by $\mathcal{R} \triangleq\left\{\mathbf{E} \in C^{n},\left\|E_{i}\right\| \geq E_{0}-\alpha\right\}, \forall i=1, \ldots, n$, where $\frac{E_{0}}{2} \leq \alpha<E_{0}-\sqrt{\left\|\mathbf{Z S} \mathbf{S}^{*}\right\|}$. Also, let $\bar{c}$ be a real constant defined as $\bar{c}=\left\|\mathbf{Z \mathbf { S } ^ { * }}\right\| /\left(E_{0}-\alpha\right)^{2}$. Observe that by construction $\left(E_{0}-\alpha\right)^{2}>\| \mathbf{Z \mathbf { S } ^ { * } \|}$ and, as consequence, $\bar{c}<1$. Also, notice that (4) can be written in the matrix form as follows.

$$
\boldsymbol{\Phi}\left(\mathbf{E}^{(k)}\right)=\mathbf{E}_{\mathbf{0}}-\mathbf{Z} \mathbf{S}^{*} \mathbf{K}^{*(k)}
$$

where $\mathbf{K}$ is a $n \times 1$ vector with elements given by reciprocals of complex voltage iterates at iteration $k$ and $\mathbf{E}_{\mathbf{0}}$ is a $n \times 1$ vector with entries equal to $E_{0}$.

Given a complex voltage $\mathbf{E}^{(k)}$ in $\mathcal{R}$, the complex voltages obtained throughout update rule can be analyzed as follows.

$$
\left\|\mathbf{\Phi}\left(\mathbf{E}^{(k)}\right)-\mathbf{E}_{0}\right\|=\left\|\mathbf{Z} \mathbf{S}^{*} \mathbf{K}^{*(k)}\right\| \leq\left\|\mathbf{Z S ^ { * } \|}\right\| \mathbf{K}^{*(k)} \|<\frac{\left\|\mathbf{Z} \mathbf{S}^{*}\right\|}{\left(E_{0}-\alpha\right)}<\left(E_{0}-\alpha\right) \leq \alpha
$$

Consequently, if $\mathbf{E}^{(k)} \in \mathcal{R}$ then $\mathbf{E}^{(k+1)}=\boldsymbol{\Phi}\left(\mathbf{E}^{(k)}\right)$ belongs to an open ball (in $C^{n}$ ) centered in $\mathbf{E}_{0}$ and a radius equal to $\alpha, \forall k=0, \ldots, N_{\text {iter }}$. Therefore, $\boldsymbol{\Phi}\left(\mathbf{E}^{(k)}\right)$ belongs to $\mathcal{R}$ and the inequality below holds $\forall k$. 


$$
0 \leq\left\|\mathbf{D}^{(k+1)}\right\|=\left\|\mathbf{Z} \mathbf{S}^{*} \mathbf{F}^{(k+1)}\right\| \leq\left\|\mathbf{Z} \mathbf{S}^{*}\right\|\left\|\mathbf{F}^{(k+1)}\right\| \leq \frac{\left\|\mathbf{Z} \mathbf{S}^{*}\right\|}{\left(E_{0}-\alpha\right)^{2}}=\bar{c}
$$

By using (10) and (12), we obtain that $0 \leq c^{(k+1)} \leq \bar{c}<1, \forall k$, as well as

$$
\left\|\boldsymbol{\Delta} \boldsymbol{\Phi}^{(k+1)}\right\| \leq \bar{c}\left\|\boldsymbol{\Delta} \mathbf{E}^{(k+1)}\right\|
$$

By construction, the function $\Phi: \mathcal{R} \rightarrow \mathcal{R}$ is a contraction mapping (in $\mathcal{R}$ ) with contraction constant $\bar{c}$ and unique fixed-point. In fact, suppose $\mathbf{E}^{\star}$ and $\mathbf{E}^{\star \star}$ are two different elements of $\mathcal{R}$, then

$$
\left\|\boldsymbol{\Phi}\left(\mathbf{E}^{\star}\right)-\mathbf{\Phi}\left(\mathbf{E}^{\star \star}\right)\right\| \leq \bar{c}\left\|\mathbf{E}^{\star}-\mathbf{E}^{\star \star}\right\|
$$

Suppose now that $\mathbf{E}^{\star}$ and $\mathbf{E}^{\star \star}$ are both load flow solutions. Then, by the hypothesis we obtain

$$
\left\|\mathbf{E}^{\star}-\mathbf{E}^{\star \star}\right\|=\left\|\mathbf{\Phi}\left(\mathbf{E}^{\star}\right)-\mathbf{\Phi}\left(\mathbf{E}^{\star \star}\right)\right\| \leq \bar{c}\left\|\mathbf{E}^{\star}-\mathbf{E}^{\star \star}\right\|
$$

If $\mathbf{E}^{\star} \neq \mathbf{E}^{\star \star}$ then $\left\|\mathbf{E}^{\star}-\mathbf{E}^{\star \star}\right\| \neq 0$. Hence, we can divide the inequality above by $\left\|\mathbf{E}^{\star}-\mathbf{E}^{\star \star}\right\|$ obtaining that $\bar{c} \geq 1$, which is an inconsistent conclusion. Therefore we have that $\mathbf{E}^{\star}=\mathbf{E}^{\star \star}$, i.e., the existence and uniqueness of the load flow feasible solution has been verified, limited to conditions related to voltage magnitude at the substation bus, network and loading.

\subsection{Power Mismatch Convergence}

Using the contraction mapping theorem, it is verified that since $\Phi: \mathcal{R} \rightarrow \mathcal{R}$ is a contraction mapping (in $\mathcal{R}$ ), the sequence of iterates $\left\{\mathbf{E}^{(0)}, \boldsymbol{\Phi}\left(\mathbf{E}^{(0)}\right), \boldsymbol{\Phi}\left(\boldsymbol{\Phi}\left(\mathbf{E}^{(0)}\right)\right), \ldots\right\}$ converges to the load flow feasible solution, $\forall \mathbf{E}_{0} \in \mathcal{R}$. The algorithm presents a geometric convergence towards the solution with a geometric rate evaluated by $c^{(k+1)}$. In addition, since $c^{(k+1)}$ depends upon system loading, the convergence characteristic is also dependent on loading.

Now, let $\Delta S_{i}^{(k)}$ be the complex power mismatch at bus $i$ and iteration $k$. In addition, let $y_{i}$ be the series line admittance upstream bus $i$. Notice that $\Delta S_{i}^{(k)}=E_{i}^{(k)}\left(E_{i}^{*(k)}-E_{u_{i}}^{*(k)}\right) y_{i}^{*}+E_{i}^{(k)} \sum_{r \in \Delta_{i}} \frac{S_{r}}{E_{r}}+S_{i}$, and by conjugating this expression we have

$$
\Delta S_{i}^{*(k)}=E_{i}^{*(k)} y_{i}\left(E_{i}^{(k)}-E_{u_{i}}^{(k)}+z_{i} \sum_{r \in \Lambda_{i}} \frac{S_{r}^{*}}{E_{r}^{*(k)}}\right)
$$

By adding the null element $E_{i}^{*(k)} y_{i}\left(\phi_{u_{i}}^{(k)}-\phi_{u_{i}}^{(k)}\right)$ in (15) we have

$$
\begin{aligned}
\Delta S_{i}^{*(k)} & =E_{i}^{(k) *} y_{i}\left(E_{i}^{(k)}-\phi_{u_{i}}^{(k)}+z_{i} \sum_{r \in \Lambda_{i}} \frac{S_{r}^{*}}{E_{r}^{*(k)}}\right)+E_{i}^{*(k)} y_{i}\left(\phi_{u_{i}}^{(k)}-E_{u_{i}}^{(k)}\right) \\
& =E_{i}^{*(k)} y_{i}\left(E_{i}^{(k)}-\phi_{i}^{(k)}\right)-E_{i}^{*(k)} y_{i}\left(E_{u_{i}}^{(k)}-\phi_{u_{i}}^{(k)}\right)
\end{aligned}
$$

Assuming $E_{i}^{*(k)} y_{i} \neq 0, \forall i=1, \ldots, n$, then

$$
\lim _{k \rightarrow \infty}\left\|\phi_{i}\left(E_{i}^{(k)}\right)-E_{i}^{(k)}\right\|=0 \Longleftrightarrow \lim _{k \rightarrow \infty}\left\|\Delta S_{i}^{(k)}\right\|=0, \forall i
$$

which indicates that voltages converge if and only if power mismatches converge to zero.

\subsection{Dependence of Initial Estimate}

A flat start or a solution estimate must be specified in the beginning of the load flow algorithm. Hence, it is important to highlight that the load flow update rule will be a contraction in a trajectory where $c<1$, and thus it will converge towards the solution if an initial estimate is chosen in a region where the contraction mapping theorem is valid. Region $\mathcal{R}$ was chosen to ease the deductions, though in theory other regions could be set to prove the same mathematical results.

Evaluation of the Forward-Backward Sweep Load Flow Method using ... (Diego Issicaba and Jorge Coelho) 
Therefore, it is noteworthy to mention that other uncommon initial estimates might lead to the solution as well. For instance, if quite high voltages are chosen as initial estimate, contraction should lead voltages at the first iteration to be located into an open ball centered in $E_{0}$ with radius $\alpha$. In fact, high voltages lead to reduced currents in the first iteration, causing voltage iterates to be close to $E_{0}$. On the other hand, if very low voltages are chosen as initial estimates, voltage errors might increase considerably leading the complex iterates into region $\mathcal{R}$. Therefore, the load flow method is shown to be robust to a large variety of initial estimates.

\subsection{Error Boundaries}

Since $\Phi: \mathcal{R} \rightarrow \mathcal{R}$ is a contraction mapping (in $\mathcal{R}$ ), with contraction constant $\bar{c}$, the following relation holds by the contraction mapping theorem

$$
\begin{gathered}
\left\|\mathbf{E}^{\propto}-\mathbf{E}^{(k)}\right\| \leq \frac{\bar{c}^{k}}{1-\bar{c}}\left\|\mathbf{\Phi}\left(\mathbf{E}^{(0)}\right)-\mathbf{E}^{(0)}\right\| \\
\left\|\mathbf{E}^{\propto}-\mathbf{E}^{(k)}\right\| \leq \frac{\bar{c}}{1-\bar{c}}\left\|\mathbf{E}^{(k)}-\mathbf{E}^{(k-1)}\right\|
\end{gathered}
$$

Therefore, by using (16) and (17) we can evaluate boundaries for the error given by the difference between the computed iterate value and the load flow solution.

\section{NUMERICAL RESULTS}

This section presents some numerical load flow analysis to validate the theoretical results deduced in the paper. The convergence criterion was chosen to be the maximum absolute difference between subsequent voltage iterates, with tolerance given by $1 \mathrm{E}-06$.

\subsection{A Two Bus Case Study}

Consider a two bus distribution feeder with substation bus voltage of $11 \mathrm{kV}$ as well as line parameter and complex load given by $1.35309+j 1.32349 \Omega$ and $5+j 3$ MVA, respectively. From the theoretical basis presented in this paper, convergence is assured and the update rule is a contraction inside region $\mathcal{R} \triangleq\left\{E_{1} \in C,\left\|E_{1}\right\| \geq E_{0}-\alpha\right\}$, for any $\alpha$ such that $0.50000 \leq \alpha<0.69800$ pu. Without loss of generality, we have chosen $\alpha$ to be 0.60000 pu leading to a contraction constant $\bar{c}$ of 0.57007 . The last iterate was considered the solution for the sake of error computation.

Table 1(a) shows the real and imaginary parts of voltage iterates, contraction iterates, error values and error boundaries computed using (17), assuming the uncommon initial estimate of $E_{1}^{(0)}=4.00 \angle 0^{\circ}$ pu. Once $E_{1}^{(0)}$ belongs to $\mathcal{R}$, contraction mapping was assigned from the very first iteration, and error boundaries indeed limited the iterate errors. As expected, contraction iterates did not exceed the contraction constant $\bar{c}$, i.e. $0<c^{(k)} \leq \bar{c}=0.57007<1$.

Table 1. Two bus case study

(a) $E_{1}^{(0)}=4.00 \angle 0^{\circ} \mathrm{pu}$

\begin{tabular}{cccccc}
\hline$k$ & $e_{1}(\mathrm{pu})$ & $f_{1}(\mathrm{pu})$ & $c^{(k)}$ & Error & Boundary \\
\hline 0 & 4.00000 & 0.00000 & - & $3.09 \mathrm{E}+00$ & - \\
1 & 0.97782 & -0.00529 & 0.02332 & $7.84 \mathrm{E}-02$ & $9.31 \mathrm{E}+00$ \\
2 & 0.90915 & -0.02113 & 0.10257 & $8.11 \mathrm{E}-03$ & $2.17 \mathrm{E}-01$ \\
3 & 0.90192 & -0.02098 & 0.11118 & $9.03 \mathrm{E}-04$ & $2.23 \mathrm{E}-02$ \\
4 & 0.90113 & -0.02114 & 0.11216 & $1.01 \mathrm{E}-04$ & $2.48 \mathrm{E}-03$ \\
5 & 0.90104 & -0.02114 & 0.11227 & $1.14 \mathrm{E}-05$ & $2.78 \mathrm{E}-04$ \\
6 & 0.90103 & -0.02114 & 0.11228 & $1.26 \mathrm{E}-06$ & $3.12 \mathrm{E}-05$ \\
7 & 0.90103 & -0.02114 & 0.11229 & $1.28 \mathrm{E}-07$ & $3.50 \mathrm{E}-06$ \\
8 & 0.90103 & -0.02114 & 0.11229 & - & $3.93 \mathrm{E}-07$
\end{tabular}

\begin{tabular}{cccccc}
\hline$k$ & $e_{1}(\mathrm{pu})$ & $f_{1}(\mathrm{pu})$ & $c^{(k)}$ & Error & Boundary \\
\hline 0 & 0.02000 & 0.00000 & - & $8.81 \mathrm{E}-01$ & $0.00 \mathrm{E}+00$ \\
1 & -3.43633 & -1.05710 & 1.26849 & $4.46 \mathrm{E}+00$ & $1.11 \mathrm{E}+01$ \\
2 & 1.02186 & 0.01288 & 0.02483 & $1.26 \mathrm{E}-01$ & $1.41 \mathrm{E}+01$ \\
3 & 0.91345 & -0.02178 & 0.09768 & $1.24 \mathrm{E}-02$ & $3.51 \mathrm{E}-01$ \\
4 & 0.90237 & -0.02082 & 0.11060 & $1.38 \mathrm{E}-03$ & $3.43 \mathrm{E}-02$ \\
5 & 0.90119 & -0.02115 & 0.11210 & $1.54 \mathrm{E}-04$ & $3.79 \mathrm{E}-03$ \\
6 & 0.90105 & -0.02114 & 0.11226 & $1.73 \mathrm{E}-05$ & $4.25 \mathrm{E}-04$ \\
7 & 0.90103 & -0.02114 & 0.11228 & $1.92 \mathrm{E}-06$ & $4.77 \mathrm{E}-05$ \\
8 & 0.90103 & -0.02114 & 0.11229 & $1.95 \mathrm{E}-07$ & $5.36 \mathrm{E}-06$ \\
9 & 0.90103 & -0.02114 & 0.11229 & - & $6.01 \mathrm{E}-07$ \\
\hline
\end{tabular}

Alternatively, the near zero initial estimate of $E_{1}^{(0)}=0.02 \angle 0^{\circ}$ pu was chosen. Voltage and contraction iterates obtained in this analysis are shown in Table 1(b). In this case, one can notice that even by choosing a nonrealistic solution as initial estimate, the algorithm converged to the feasible solution. In fact, the contraction iterate $c^{(k)}$ is greater than the unit near the initial estimate, where the update rule is not a contraction mapping

IJECE Vol. 6, No. 6, December 2016: 3229 - 3237 
(dilation). As a consequence, voltage mismatches increased and voltage iterates moved towards region $\mathcal{R}$, where convergence is guaranteed by the mathematical results.

\subsection{A 27-bus Case Study}

The load flow algorithm was applied to the 27-bus system given in [21]. The mathematical analysis showed that convergence is assured and the update rule is a contraction into the region $\mathcal{R} \triangleq\left\{\mathbf{E} \in C^{27},\left\|E_{i}\right\| \geq E_{0}-\alpha\right.$, $\forall i=1, \ldots, 27\}$, for any $\alpha$ such that $0.50000 \leq \alpha<0.71270$. Similar to the previous case study, an $\alpha$ value of 0.60000 was chosen and the last iterate was considered as solution for error computation purposes. The contraction constant $\bar{c}$ is 0.51580 for this case.

The initial estimate was chosen to be $E_{i}^{(0)}=0.05 \angle 120.32^{\circ} \mathrm{pu}, \forall i=1, \ldots, 27$. Table 3 shows the iterates obtained in this simulation. Also, the first two voltage iterates are illustrated in a level curve of the convergence region for bus 25 in Fig. 2.

Table 2. The 27-bus case study (bus 25). Initial estimate: $E_{i}^{(0)}=0.05 \angle 120.32^{\circ} \mathrm{pu}$.

\begin{tabular}{cccccc}
\hline$k$ & $e_{25}(\mathrm{pu})$ & $f_{25}(\mathrm{pu})$ & $c^{(k)}$ & Error & Boundary \\
\hline 0 & -0.02524 & 0.04316 & - & $1.01 \mathrm{E}-00$ & - \\
1 & 1.51846 & 1.56655 & 0.83438 & $1.70 \mathrm{E}-00$ & $2.31 \mathrm{E}-00$ \\
2 & 0.97564 & -0.03361 & 0.04262 & $6.54 \mathrm{E}-02$ & $1.80 \mathrm{E}-00$ \\
3 & 0.91703 & -0.01505 & 0.09096 & $5.19 \mathrm{E}-03$ & $6.55 \mathrm{E}-02$ \\
4 & 0.91273 & -0.01732 & 0.09637 & $4.30 \mathrm{E}-04$ & $5.18 \mathrm{E}-03$ \\
5 & 0.91234 & -0.01720 & 0.09679 & $3.56 \mathrm{E}-05$ & $4.29 \mathrm{E}-04$ \\
6 & 0.91231 & -0.01721 & 0.09683 & $2.94 \mathrm{E}-06$ & $3.56 \mathrm{E}-05$ \\
7 & 0.91231 & -0.01721 & 0.09683 & $2.29 \mathrm{E}-07$ & $2.95 \mathrm{E}-06$ \\
8 & 0.91231 & -0.01721 & 0.09683 & - & $2.44 \mathrm{E}-07$ \\
\hline
\end{tabular}

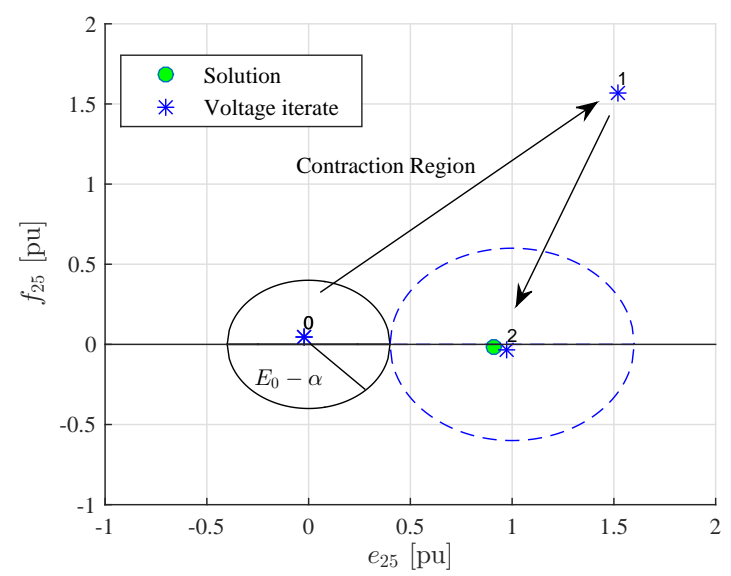

Figure 2. Three voltage iterates for the 27-bus case study (bus 25). Initial estimate $E_{i}^{(0)}=0.05 \angle 120.32^{\mathrm{o}} \mathrm{pu}$.

As expected, since the initial estimate has low voltage entries the voltage mismatches increased causing the voltage iterates to be placed in $\mathcal{R}$, where the convergence is guaranteed by the mathematical analysis. Also, the contraction iterates did not exceed the contraction constant in $\mathcal{R}$ and the error boundaries indeed limited the iterate error values.

\section{CONCLUSIONS}

This paper evaluates the forward-backward sweep load flow method to distribution system analysis using fixed-point concepts and the contraction mapping theorem. The convergence of the method is proven given certain conditions related to substation bus voltage, network data and system loading. The existence and uniqueness of the feasible load flow solution is also evaluated, subjected to the same conditions. Furthermore, boundaries for error values per iteration between iterates and solution are obtained. It was shown that the 
algorithm exhibits a geometric convergence towards solution. Also, the algorithm is robust to a large variety of initial estimates.

All the mathematical results are validated with load flow simulations. Future works are envisioned to extend to developed analysis with regard to three-phase distribution networks, weakly-meshed distribution networks, voltage dependent load models and distributed generators.

\section{ACKNOWLEDGMENTS}

The authors would like to acknowledge the financial, technical and human support of the CNPq, CAPES, INESC Porto and INESC P\&D Brasil.

\section{REFERENCES}

[1] D. Issicaba, A. J. S. Costa, and J. L. Colombo, "Real-time monitoring of points of common coupling in distribution systems through state estimation and geometric tests," IEEE Transactions on Smart Grid, vol. 7, no. 1, pp. 9-18, Jan. 2016.

[2] H. Marefatjou and M. Sarvi, "Distributed generation allocation to improve steady state voltage stability of distribution networks using imperialist competitive algorithm," International Journal of Applied Power Engineering (IJAPE), vol. 2, no. 1, pp. 15-26, 2013.

[3] D. Issicaba and J. Coelho, "Rotational load flow method for radial distribution systems," International Journal of Electrical and Computer Engineering (IJEPE), vol. 6, no. 3, 2016.

[4] A. J. Korsak, "On the question of uniqueness of stable load flow solutions," IEEE Transactions on Power Apparatus and Systems, vol. PAS-91, no. 3, pp. 1093-1100, May 1972.

[5] B. K. Johnson, "Extraneous and false load flow solutions," IEEE Transactions on Power Apparatus and Systems, vol. PAS-96, no. 2, pp. 524-534, March/April 1977.

[6] C. Tavora and O. Smith, "Equilibrium analysis of power systems," IEEE Transactions on Power Apparatus and Systems, vol. PAS-91, no. 3, pp. 1131-1137, May 1972.

[7] J. Thorp, D. Schulz, and M. Ilic-Spong, "Reactive power-voltage problem: Conditions for existence of solution and localized disturbance propagation," International Journal of Electrical Power and Systems, vol. 8, no. 2, pp. 66-74, April 1986.

[8] H. D. Chiang and M. E. Baran, "On the existence and uniqueness of load flow solution for radial distribution networks," IEEE Transactions on Circuits and Systems, vol. 37, no. 3, pp. 410-416, March 1990.

[9] J. F. Chen and W. M. Wang, "Uniqueness of the feasible voltage solutions for radial power networks," in IEEE Region 10 International Conference on Microeletronics and VLSI, TENCON's 95, Novemeer 1995, pp. 351-354.

[10] M. E. Baran and F. F. Wu, "Optimal sizing of capacitor placed on a radial distribution system," IEEE Transaction on Power Delivery, vol. 4, no. 1, pp. 735-743, January 1989.

[11] _ _ "Network reconfiguration in distribution systems for loss reduction and load balancing," IEEE Transactions on Power Delivery, vol. 4, no. 2, pp. 1401-1407, April 1989.

[12] K. N. Miu and H. D. Chiang, "Existence, uniqueness, and monotonic properties of the feasible power flow solution for radial three-phase distribution networks," IEEE Transactions on Circuits and Systems I:Fundamental Theory and Applications, vol. 47, no. 10, pp. 1502-1514, October 2000.

[13] J. Meisel and R. D. Barnard, "Application of fixed-point techniques to load flow studies," IEEE Transactions on Power and Apparatus and Systems, vol. PAS-89, no. 1, pp. 138-1970, January 1970.

[14] K. Ganesan, E. Moore, and W. J. Vetter, "On convergence of newton's method for load flow problem," International Journal of Numerical Methods in Engineering, vol. 3, pp. 325-336, 1973.

[15] F. F. Wu, "Theoretical study of the convergence of the fast load flow method," IEEE Transactions on Power Apparatus and Systems, vol. PAS-96, no. 1, pp. 268-275, January/February 1977.

[16] D. Shirmohammadi, H. W. Hong, A. Semlyen, and G. X. Luo, "A compensation-based power flow method for weakly meshed distribution and transmission networks," IEEE Transactions on Power Systems, vol. 3, no. 2, pp. 753-762, May 1988.

[17] R. P. Broadwater, A. Chandrasekaran, C. T. Huddleston, and A. H. Khan, "Power flow analysis of unbalanced multiphase radial distribution systems," Electric Power Systems Research, vol. 14, no. 1, pp. 23-33, February 1988.

[18] R. G. Cespedes, "New method for the analysis of distribution networks," IEEE Transaction on Power Delivery, vol. 5, no. 1, pp. 391-396, January 1990.

IJECE Vol. 6, No. 6, December 2016: 3229 - 3237 
[19] E. Bompard, E. Carpaneto, G. Chicco, and R. Napoli, "Convergence of the backward-forward sweep method for the load flow analysis of radial distribution systems," Electrical Power \& Energy Systems, vol. 22, no. 7, pp. 521-530, October 2000.

[20] W. Rudin, Principles of Mathematical Analysis, 3rd ed. New York, USA: McGraw-Hill, 1976.

[21] D. Das, H. S. Nagi, and D. P. Kothari, "Novel method for solving radial distribution networks," in IEE Proceedings on Generation, Transmission and Distribution, vol. 141, no. 4, July 1994, pp. 291-298.

\section{BIOGRAPHIES OF AUTHORS}

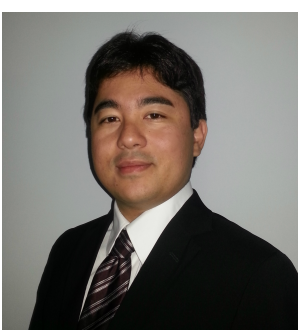

Diego Issicaba received the B.S. and M.S. degrees in Electrical Engineering from the Federal University of Santa Catarina (UFSC), Santa Catarina, Brazil, in 2006 and 2008, respectively. Furthermore, he received the Ph.D. degree on Sustainable Energy Systems, under the MIT Doctoral Program, from the Faculty of Engineering of the University of Porto, Portugal. His research interests involve smart grids, mutiagent systems, distributed generation and distribution systems. He is currently a full Professor at Federal University of Technology - Parana (UTFPR), Associate Researcher and Coordinator of the Research Area on Energy and Management of INESC P\&D Brasil.

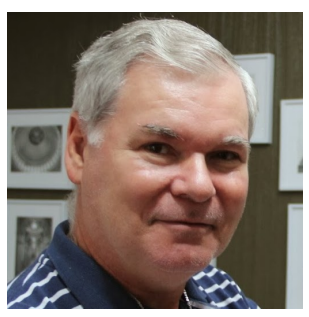

Jorge Coelho received the B.S. and M.S. degrees in electrical engineering from the Federal University of Santa Catarina, Brazil, in 1977 and 1980, respectively. In 1990, he received the Ph.D. degree in electrical engineering from the Catholic University of Rio de Janeiro, Brazil. He is a Professor of the Department of Electrical Engineering at the Federal University of Santa Catarina, Brazil, since March 1978. His research interests include distribution systems expansion and operation planning, power systems reliability, probabilistic methods applied to power systems, and power quality. 